\title{
Twenty-five-year changing pattern of gonococcal antimicrobial susceptibility in Shanghai: surveillance and its impact on treatment guidelines
}

Wei-Ming Gu${ }^{1 *}$, Yue Chen², Yang Yang ${ }^{1}$, Lei Wu' ${ }^{1}$, Wei-Zhong Hu${ }^{1}$ and Yue-Lan Jin ${ }^{1}$

\begin{abstract}
Background: Antimicrobial resistance of Neisseria gonorrhoeae is a serious health problem in China. Gonococcal antimicrobial susceptibility has been monitored in Shanghai since 1988. In this study, we examined the changing pattern of gonococcal antimicrobial susceptibility based on data from N. gonorrhoeae isolates collected over the past 25 years.
\end{abstract}

Methods: Approximately 100-200 isolates each year (1988-2013) were tested for their susceptibility to penicillin (PEN), tetracycline (TET), ciprofloxacin (CIP), ceftriaxone (CRO) and spectinomycin (SPT), using the agar dilution method. Plasmid-mediated N. gonorrhoeae antimicrobial resistance, comprising penicillinase-producing N. gonorrhoeae (presumed PPNG) and high-level tetracycline resistance N. gonorrhoeae (presumed TRNG), were also determined. Breakpoints for susceptibilities followed those described by the Clinical and Laboratory Standard Institute and the European Committee on Antimicrobial Susceptibility Testing.

Results: A high proportion of isolates were resistant to PEN, TET and CIP, ranging from less than 20\% at the beginning of the survey, increasing in the late 1990s and reaching over $90 \%$ in recent years. The proportion of isolates exhibiting plasmid-mediated resistance exceeded 38\% for presumed PPNG and 20\% for presumed TRNG in recent years. The proportion of CRO nonsusceptible isolates (MIC $\geq 0.125 \mathrm{mg} / \mathrm{L}$ ) ranged from $7 \%$ to $13 \%$ in most of the study years. Almost all isolates were susceptible to SPT. The SPT MIC 90 was 16-32 mg/L for 2008-2013. The proportion of CRO nonsusceptible-associated multiple-drug-resistant (MDR) isolates was over $5 \%$ in most of the study years.

Conclusions: N. gonorrhoeae isolates in Shanghai were resistant to PEN, TET and CIP. Furthermore, CRO nonsusceptible and MDR isolates were prevalent. N. gonorrhoeae isolates were also found to be susceptible to SPT. It is recommended that the CRO dose be increased from currently recommended $250 \mathrm{mg}$ to $500 \mathrm{mg}$ and that SPT be an alternative in treating urogenital gonorrhea. Our findings highlight the importance of both regional and national surveillance programs for the prompt modification of treatment guidelines, vital in responding to the changing pattern of gonococcal antimicrobial susceptibility.

Keywords: Neisseria gonorrhoeae, Antimicrobial susceptibility, Multiple drug resistance, Ceftriaxone, Spectinomycin

\footnotetext{
* Correspondence: weiming_gu2003@aliyun.com

'Shanghai Skin Disease Hospital, Shanghai, China

Full list of author information is available at the end of the article
} 


\section{Background}

Neisseria gonorrhoeae, a Gram-negative bacterium, is responsible for the sexually transmitted disease gonorrhea. In 2008, over 106 million new cases of $N$. gonorrhoeae were reported worldwide [1]. In China, infectious disease surveillance reports more than 100,000 new gonorrhea cases each year. In 2012, there were 95,263 gonorrhea cases reported in China with an incidence rate of 7.07 per 100,000 population. Gonorrhea ranked sixth in the most frequently reported infectious diseases in China [2]. There are currently no vaccines available for $N$. gonorrhoeae [3]. Antimicrobial treatment is essential to control this infectious disease. However, antimicrobial resistance (AMR) in $N$. gonorrhoeae is emerging as a significant public health problem worldwide $[4,5]$. In most areas of the world, $N$. gonorrhoeae has developed resistance to sulfonamides, penicillins, tetracyclines, macrolides and fluoroquinolones [6]. Decreased susceptibility, sporadic resistant isolates and clinical treatment failure to oral or parental thirdgeneration cephalosporins have also been observed [7-10]. Moreover, extensive/multiple-drug-resistant (MDR) $N$. gonorrhoeae isolates are commonly observed in various areas of the world $[8,10]$.

In China, the burden of gonococcal penicillin (PEN) and tetracycline (TET) resistance reached high levels [11-13]. In response, fluoroquinolones were introduced as a treatment option. Since 1995, orally administered ciprofloxacin (CIP) has been recommended for the treatment of gonorrhea in China. However, as a result, the proportion of CIP resistance drastically increased from 15\% in 1996 to nearly $100 \%$ in the mid-2000s in China [11-13]. The use of CIP for the treatment of gonorrhea has since been terminated in China [14,15]. Since 2007, ceftriaxone (CRO) has been recommended as the first line drug for the treatment of gonorrhea in China [14]. However, in Shanghai CRO has been used since 2000, and the presence and spread of gonococcal isolates with nonsusceptibility to CRO (minimal inhibitory concentration $(\mathrm{MIC})=0.125-0.25 \mathrm{mg} / \mathrm{L}$ ) was reported in $11.9 \%$ of isolates in 2005 and $19.7 \%$ of isolates in this city in 2008 [11,12].

In this study, we used the data of the Shanghai Gonococcal Antimicrobial Susceptibility Surveillance Program from $N$. gonorrhoeae isolates collected over the past 25 years to analyze trends in AMR development in Shanghai. The AMR data were aligned with the timeline of treatment guidelines for gonorrhea in China. We found that $N$. gonorrhoeae isolates in Shanghai were resistant to PEN, TET and CIP, which were historically used for the treatment of gonorrhea in this region. Plasmid-mediated N. gonorrhoeae antimicrobial resistance has been prevalent in the past decades. High proportions of $N$. gonorrhoeae isolates are nonsusceptible to the currently recommended treatment regime of CRO (MIC $\geq 0.125$ ). In Shanghai, MDR associated with the $\mathrm{CRO}$ nonsusceptible phenotype was prevalent in $N$. gonorrhoeae isolates. Our report indicates the usefulness of regional surveillance data in the modification of treatment guidelines and provides insights that will be important in future strategic planning for the treatment of gonorrhea.

\section{Methods}

\section{Collection of $N$. gonorrhoeae isolates}

The Shanghai Gonococcal Antimicrobial Susceptibility Surveillance Program was established in 1988 and has been led by the Shanghai Skin Disease Hospital (SSDH), Shanghai, China. The number of gonorrhea cases reported by the SSDH was approximately $14-16 \%$ of the reported cases from the entire Shanghai city over the years [16]. The number of gonococcal isolates tested for antimicrobial susceptibility accounted for about $10 \%$ of the SSDH reported cases or $2.1 \%$ of reported cases in Shanghai in 2012. All of the surveyed N. gonorrhoeae isolates were from the Sexually Transmitted Infection Clinic of the SSDH.

Each year in the SSDH, approximately 100-200 N. gonorrhoeae isolates were collected from individual patients for antimicrobial susceptibility testing. The first 10-20N. gonorrhoeae isolates each month were collected from patients with culture positive gonorrhea [12]. During the period from 1988 to 2000, N. gonorrhoeae isolates were collected from both male and female patients, and during the period from 2001 to 2013, N. gonorrhoeae isolates were only collected from male patients. N. gonorrhoeae was isolated and cultured as previously described [12].

Gonococcal isolates were collected as part of standard patient care, and ethics approval was not required for their use.

\section{Determination of the minimal inhibitory concentrations (MIC)}

The antimicrobial susceptibility of $N$. gonorrhoeae isolates was determined using the agar dilution method [17] at the Laboratory Diagnostic Centre of the SSDH. WHO reference strains A, B, C, D and E were used in susceptibility tests [12]. Five antimicrobial agents were tested against $N$. gonorrhoeae isolates: PEN (0.002-32.0 mg/L), TET (0.002$32.0 \mathrm{mg} / \mathrm{L})$, CIP (0.002-32.0 mg/L), spectinomycin (SPT; 2.0-128.0 mg/L) and CRO (0.002-2.0 mg/L). Antimicrobial agents were provided by the China GASP at the National Center for Sexually Transmitted Disease Control, China (1988-2002) or purchased from Sigma-Aldrich (Distributor: the Shanghai ANPEL Scientific Instrument Co. Ltd., Shanghai, China). The MIC testing was performed on GC agar base supplemented with $1 \%$ IsoVitalex as previously described [12]. Each MIC determination was performed in duplicate. Gonococcal isolates with inconsistent CRO MICs between the duplicates were re-tested. 


\section{Determination of plasmid-mediated resistance in $\mathbf{N}$. gonorrhoeae isolates}

Beta-lactamase producing $N$. gonorrhoeae (PPNG) isolates were determined using the chromogenic cephalosporin test before 2003 and the nitrocefin test in 2003-2013 (Oxoid; distributed by GuangZhou LOSO Science Ltd.) [12]. All $N$. gonorrhoeae isolates were tested for $\beta$-lactamase production. Plasmid-mediated tetracycline-resistant $N$. gonorrhoeae (TRNG) isolates were defined as those having a TET MIC of $\geq 16.0 \mathrm{mg} / \mathrm{L}$. PP/TRNG isolates were defined as those isolates that were $\beta$-lactamase positive and had a TET MIC of $\geq 16 \mathrm{mg} / \mathrm{L}$. Therefore, the characterizations for presumed PPNG and TRNG were solely based on the detection of $\beta$-lactamase and the TET MIC, respectively.

\section{Criteria for resistant phenotypes of $N$. gonorrhoeae}

Breakpoints for the susceptibilities to PEN, TET, CIP and SPT were those described by the Clinical Laboratory Standards Institute (CLSI) [17]. The classifications of resistance phenotypes included $\mathrm{PEN}$ resistance $(\mathrm{MIC} \geq$ $2.0 \mathrm{mg} / \mathrm{L}$ ), TET resistance (MIC $\geq 2.0 \mathrm{mg} / \mathrm{L})$, CIP resistance $(\mathrm{MIC} \geq 1.0 \mathrm{mg} / \mathrm{L})$ and $\mathrm{SPT}$ resistance $(\mathrm{MIC} \geq$ $128.0 \mathrm{mg} / \mathrm{L})$. Chromosomally-mediated resistant $N$. gonorrhoeae (CMRNG) isolates exhibited a PEN MIC of $\geq 2.0 \mathrm{mg} / \mathrm{L}$, a TET $\mathrm{MIC}$ of $\geq 2.0 \mathrm{mg} / \mathrm{L}$ and were not classified as PPNG or TRNG. For CRO susceptibility, the European Committee on Antimicrobial Susceptibility Testing (EUCAST) breakpoint was followed (nonsusceptible, MIC $\geq 0.125 \mathrm{mg} / \mathrm{L}$ ) [18]. In this study, the susceptibilities to $\mathrm{CRO}$ in $N$. gonorrhoeae were further categorized as susceptible $(\mathrm{MIC} \leq 0.03 \mathrm{mg} / \mathrm{L})$, reduced susceptibility $(\mathrm{MIC}=0.06)$ and nonsusceptible $(\mathrm{MIC} \geq$ $0.125 \mathrm{mg} / \mathrm{L})$ [11]. The MDR phenotypes of $N$. gonorrhoeae were defined as various combinations of the above resistant phenotypes (Table 1).

\section{Results}

N. gonorrhoeae has become less susceptible to CRO

During the period from 1995 to 2013, 3153 N. gonorrhoeae isolates were examined for their susceptibility to CRO (Table 2). Overall, $71.90 \%$ isolates were susceptible to CRO (MIC $\leq 0.03 \mathrm{mg} / \mathrm{L}) ; 20.30 \%$ had a reduced susceptibility phenotype $(\mathrm{MIC}=0.06 \mathrm{mg} / \mathrm{L})$ and $7.80 \%$ exhibited a CRO nonsusceptible phenotype ( $\mathrm{MIC} \geq 0.125 \mathrm{mg} / \mathrm{L})$. The highest CRO MIC value for the $N$. gonorrhoeae isolates was $0.25 \mathrm{mg} / \mathrm{L}$, which accounted for $0.92 \%$ of the isolates tested (29/3153).

The proportions of $\mathrm{CRO}$ non-susceptible isolates were over or equal to $5 \%$ in all years since 1998, and were around $7 \%-13 \%$ in the last 3 years (2011 to 2013). The proportion of isolates showing reduced susceptibility to CRO ranged from $12 \%$ to $40 \%$ from 1998 to 2013. From 1998 to 2013, the $\mathrm{MIC}_{50}$ values for CRO were between

\begin{tabular}{|c|c|c|c|c|}
\hline Resistant phenotype & PEN & TET & CIP & CRO \\
\hline CMRNG & $\geq 2$ & $\geq 2$ & na & na \\
\hline PEN-TET & $\geq 2$ & $\geq 2$ & na & na \\
\hline PEN-CIP & $\geq 2$ & na & $\geq 1$ & na \\
\hline PEN-CRO & $\geq 2$ & na & na & $\geq 0.125$ \\
\hline TET-CIP & na & $\geq 2$ & $\geq 1$ & na \\
\hline TET-CRO & na & $\geq 2$ & na & $\geq 0.125$ \\
\hline CIP-CRO & na & na & $\geq 1$ & $\geq 0.125$ \\
\hline PEN-TET-CIP & $\geq 2$ & $\geq 2$ & $\geq 1$ & na \\
\hline PEN-TET-CRO & $\geq 2$ & $\geq 2$ & na & $\geq 0.125$ \\
\hline PEN-CIP-CRO & $\geq 2$ & na & $\geq 1$ & $\geq 0.125$ \\
\hline TET-CIP-CRO & na & $\geq 2$ & $\geq 1$ & $\geq 0.125$ \\
\hline PEN-TET-CIP-CRO & $\geq 2$ & $\geq 2$ & $\geq 1$ & $\geq 0.125$ \\
\hline
\end{tabular}

CMRNG: chromosomally-mediated resistant $N$. gonorrhoeae, non-penicillinaseproducing $N$. gonorrhoeae (Non-PPNG) and tetracycline MIC $<16 \mathrm{mg} / \mathrm{L}$. PEN: penicillin; TET: tetracycline; CIP: ciprofloxacin; CRO: ceftriaxone (nonsusceptible); na: not applicable; MICs are presented as $\mathrm{mg} / \mathrm{L}$; Spectinomycin (SPT) was not listed.

$0.015 \mathrm{mg} / \mathrm{L}$ and $0.06 \mathrm{mg} / \mathrm{L}$, and the $\mathrm{MIC}_{90}$ values were 0.06-0.125 mg/L (Table 2).

\section{N. gonorrhoeae is resistant to CIP, PEN and TET}

During the period from 1996 to 2013, 3036 N. gonorrhoeae isolates were examined for susceptibility to CIP. Overall, $85.30 \%$ isolates were resistant to CIP. CIP resistant $N$. gonorrhoeae accounted for $16.50 \%$ of the $N$. gonorrhoeae isolates in 1996, compared with over $85 \%$ in 1999 . CIP resistance among the isolates ranged from $88 \%$ to $100 \%$ in the period between 2000 and 2013 (Additional file 1: Figure S1). A total of $3663 N$. gonorrhoeae isolates were examined for their susceptibility to PEN. Overall, 62.00\% isolates were resistant to PEN. Proportions of PEN resistance increased from $20-40 \%$ before 1998, to $70-90 \%$ during the period from 2000 to 2013 (Additional file 1: Figure S1). During the period from 1995 to 2013, $3153 N$. gonorrhoeae isolates were examined for their susceptibility to TET. Only one concentration of TET $(16 \mathrm{mg} / \mathrm{L})$ was tested during 1998-2003 $(\mathrm{n}=1355)$, whereas the remaining $1798 N$. gonorrhoeae isolates were tested for the full range of TET concentrations. For the 1798 isolates tested during 1995-1997 and 2004-2013, 50.61\% (910/ 1798) were resistant to TET. The proportion of TET resistant isolates increased from 16\% (1995-1997) to over 40\% (2004-2012), and then further increased to $82.57 \%$ in 2013 (Additional file 1: Figure S1).

\section{Susceptibility of $\boldsymbol{N}$. gonorrhoeae isolates to SPT}

From 1995 to 2013, 3153 N. gonorrhoeae isolates were examined for their susceptibility to SPT. Only two resistant 
Table 2 Susceptibility of $\boldsymbol{N}$. gonorrhoeae isolates to ceftriaxone in Shanghai

\begin{tabular}{|c|c|c|c|c|c|c|c|c|}
\hline \multirow[t]{2}{*}{ Year } & \multirow[t]{2}{*}{$\mathbf{N}$} & \multicolumn{2}{|c|}{ Reduced* } & \multicolumn{2}{|c|}{ Nonsusceptible ${ }^{* *}$} & \multirow{2}{*}{$\begin{array}{l}\mathrm{MIC}_{50} \\
(\mathrm{mg} / \mathrm{L})\end{array}$} & \multirow{2}{*}{$\begin{array}{l}\mathrm{MIC}_{90} \\
(\mathrm{mg} / \mathrm{L})\end{array}$} & \multirow{2}{*}{$\begin{array}{l}\text { MIC range } \\
(\mathrm{mg} / \mathrm{L})\end{array}$} \\
\hline & & $\mathrm{n}$ & $\%$ & $\mathrm{n}$ & $\%$ & & & \\
\hline 1995 & 117 & 0 & 0.00 & 0 & 0.00 & 0.008 & 0.015 & $0.008-0.03$ \\
\hline 1996 & 206 & 2 & 0.97 & 2 & 0.97 & 0.008 & 0.03 & $0.002-0.25$ \\
\hline 1997 & 208 & 7 & 3.37 & 2 & 0.96 & 0.008 & 0.03 & $0.002-0.25$ \\
\hline 1998 & 180 & 29 & 16.11 & 14 & 7.78 & 0.03 & 0.06 & $0.004-0.25$ \\
\hline 1999 & 208 & 27 & 12.98 & 12 & 5.77 & 0.015 & 0.06 & $0.002-0.25$ \\
\hline 2000 & 225 & 47 & 20.89 & 15 & 6.67 & 0.03 & 0.06 & $0.004-0.25$ \\
\hline 2001 & 230 & 39 & 16.96 & 16 & 6.96 & 0.03 & 0.06 & $0.004-0.25$ \\
\hline 2002 & 210 & 30 & 14.29 & 20 & 9.52 & 0.03 & 0.06 & $0.004-0.25$ \\
\hline 2003 & 302 & 57 & 18.87 & 34 & 11.26 & 0.03 & 0.125 & $0.004-0.25$ \\
\hline 2004 & 166 & 53 & 31.93 & 20 & 12.05 & 0.03 & 0.125 & $0.004-0.25$ \\
\hline 2005 & 83 & 26 & 31.33 & 5 & 6.02 & 0.03 & 0.06 & $0.004-0.125$ \\
\hline 2006 & 128 & 51 & 39.84 & 18 & 14.06 & 0.06 & 0.125 & $0.008-0.25$ \\
\hline 2007 & 200 & 64 & 32.00 & 10 & 5.00 & 0.03 & 0.06 & $0.004-0.25$ \\
\hline 2008 & 71 & 25 & 35.21 & 14 & 19.72 & 0.06 & 0.125 & $0.008-0.125$ \\
\hline 2009 & 157 & 36 & 22.93 & 18 & 11.46 & 0.03 & 0.125 & $0.008-0.125$ \\
\hline 2010 & 119 & 34 & 28.57 & 9 & 7.56 & 0.03 & 0.06 & $0.008-0.125$ \\
\hline 2011 & 135 & 55 & 40.74 & 14 & 10.37 & 0.06 & 0.125 & $0.008-0.125$ \\
\hline 2012 & 99 & 14 & 14.14 & 7 & 7.07 & 0.03 & 0.06 & $0.004-0.125$ \\
\hline 2013 & 109 & 43 & 39.45 & 15 & 13.76 & 0.06 & 0.125 & $0.004-0.25$ \\
\hline Total & 3153 & 639 & 20.30 & 245 & 7.80 & & & \\
\hline
\end{tabular}

*: CRO MIC $=0.06 \mathrm{mg} / \mathrm{L} ;$ **: CRO MIC $\geq 0.125 \mathrm{mg} / \mathrm{L}$.

isolates $(0.06 \%, \mathrm{MIC} \geq 128 \mathrm{mg} / \mathrm{L})$ were identified, one in 1999 and the other in 2002. Overall, isolates with intermediate resistance $(\mathrm{MIC}=64 \mathrm{mg} / \mathrm{L})$ accounted for $6.76 \%$, and the related proportion of susceptible isolates was 93.18\%. During the period from 2009 to 2013, the proportion of isolates with an SPT MIC $\geq 64 \mathrm{mg} / \mathrm{L}$ was less than $5 \%$ (4.46\% in 2009, $0 \%$ in 2010, 2.22\% in 2011 and $0 \%$ in 2012 and 2013), with the SPT $\mathrm{MIC}_{50}$ ranging between $4 \mathrm{mg} / \mathrm{L}$ and $32 \mathrm{mg} / \mathrm{L}$ during this period. The majority of SPT $\mathrm{MIC}_{90}$ values were $16-32 \mathrm{mg} / \mathrm{L}$, and the values of $\mathrm{MIC}_{90}$ in 2003 and 2007 were $64 \mathrm{mg} / \mathrm{L}$ (data not shown).

\section{Plasmid-mediated and chromosomally-mediated resistant N. gonorrhoeae}

In 1995 , only $0.85 \%$ of isolates exhibited a presumed PPNG phenotype. Around 5\% of isolates were presumed PPNG from 1996 to 1998 . The proportion of presumed PPNG isolates increased rapidly from $13 \%$ in 1999 to $37 \%$ in 2000 and ranged from $37 \%$ to $57 \%$ during the period from 2001 to 2013 (Additional file 1: Figure S2). The proportion of presumed TRNG isolates was $0-1 \%$ for the period from 1995 to 1998, and increased to $7.69 \%$ and $6.67 \%$ for 1999 and 2000, respectively. During 2001-2005, the proportion of presumed TRNG was around $17 \%-23 \%$. This proportion again increased from
$30 \%$ (2006) to 56\% (2008), and remained above 30\%, from 2009 to 2013 (Additional file 1: Figure S2). There were no PP/TRNG isolates detected during the first 4 years of surveillance (1995-1998). The proportion of presumed PP/TRNG isolates was $0.48 \%$ and $1.78 \%$ for 1999 and 2000, respectively. This proportion increased rapidly to $12-14 \%$ during the period from 2001 to 2005 and remained above 15\% between 2006 and 2013, peaking at $33.8 \%$ in 2008 (Additional file 1: Figure S2).

The proportion of chromosomally-mediated resistance to PEN and TET among the 1569 CMRNG isolates from 2003 to 2013 ranged from $4.23 \%$ to $29.14 \%$. In recent years, the proportion of CMRNG isolates detected has been $15.56 \%$ (2011), 6.06\% (2012) and 23.85\% (2013).

\section{Multiple drug resistance (MDR) in N. gonorrhoeae}

An MDR phenotype for $N$. gonorrhoeae was defined as resistance to two or more antimicrobial agents (Table 1). Double-resistant phenotypes accounted for 39-68\% (PEN-TET), 61-92\% (PEN-CIP) and 43-81\% (TET-CIP) of $N$. gonorrhoeae isolates, and the triple-resistant phenotype (PEN-TET-CIP) accounted for 38-61\% of $N$. gonorrhoeae isolates during the period from 2003 to 2013 (data not shown). 
Between 2001 and 2013, the proportion of the CRO nonsusceptible phenotype combined with PEN, TET or CIP resistance fluctuated from $4.5 \%$ to $19.72 \%$ (PEN-CRO), $2.41 \%$ to $12.68 \%$ (TET-CRO) or $0 \%$ to $19.72 \%$ (CIP-CRO) (Table 3). CRO-associated triple-resistant phenotypes accounted for $2.41-12.68 \%$ (PEN-TET-CRO), 2.41-12.68\% (TET-CIP-CRO) and 2.17-19.72\% (PEN-CIP-CRO) of $N$. gonorrhoeae isolates (Table 3), and the proportion of quadruple resistant phenotypes (PEN-TET-CIP-CRO) accounted for $2.41-12.68 \%$ of $N$. gonorrhoeae isolates (Table 3).

\section{Discussion}

In China, antibiotic resistance of $N$. gonorrhoeae has become an increasing problem over the past few decades in the treatment of gonorrhea infection. Since the mid1990s, a high proportion of $N$. gonorrhoeae isolates sampled in Shanghai were resistant to PEN, TET and CIP. Plasmid-mediated resistance has been highly prevalent since the early 2000s. The proportion of $N$. gonorrhoeae isolates exhibiting a CRO nonsusceptible phenotype $(\mathrm{MIC} \geq 0.125 \mathrm{mg} / \mathrm{L})$ has been greater than $5 \%$ since 1998. In 2013, $13.76 \%$ of isolates exhibited a CRO MIC of $\geq 0.125 \mathrm{mg} / \mathrm{L}$, suggesting that the effectiveness of the currently recommended treatment regime for gonorrhea is diminishing. MDR phenotypes associated with PEN, TET and CIP resistance were prevalent. $N$. gonorrhoeae isolates exhibiting a CRO nonsusceptible phenotype associated MDR were also found to be prevalent in recent years. In Shanghai, N. gonorrhoeae isolates were found to be susceptible to SPT.

The CRO MIC of $N$. gonorrhoeae isolates has been extensively monitored. In the United States, the proportion of isolates with elevated CRO MICs $(\geq 0.125 \mathrm{mg} / \mathrm{L})$ ranged from $0.1 \%$ to $0.4 \%$ between 2006 and 2012 [19].
In Canada, $7.3 \%$ of $N$. gonorrhoeae isolates showed CRO MICs $\geq 0.125 \mathrm{mg} / \mathrm{L}$ in 2010 [20]. In England and Wales during 2007-2011, 0-0.4\% of N. gonorrhoeae isolates showed CRO MICs $\geq 0.125 \mathrm{mg} / \mathrm{L}$ [4]. In Australia, no isolates of $N$. gonorrhoeae with $\mathrm{CRO}$ MIC values $\geq$ $0.125 \mathrm{mg} / \mathrm{L}$ were reported in 2011 [21,22]. In Latin America and the Caribbean countries, $0.4 \%$ of $N$. gonorrhoeae isolates had CRO MICs $\geq 0.125 \mathrm{mg} / \mathrm{L}$ between 2007 and 2011 [23].

In the present study, we found that the proportion of $N$. gonorrhoeae isolates with CRO MICs $\geq 0.125 \mathrm{mg} / \mathrm{L}$ in Shanghai (1996-2013) greatly varied from year to year, ranging from $0 \%$ (1995) to $19.72 \%$ in 2008 , and $13.76 \%$ in 2013. Our findings were consistent with those of a survey of CRO susceptibility in Nanjing, China [24,25]. The proportion of CRO less susceptible $N$. gonorrhoeae isolates ( $\mathrm{CRO} \quad \mathrm{MIC} \geq 0.06 \mathrm{mg} / \mathrm{L}$ ) was $17.86-50.5 \%$ during the period from 1999 to 2006 in Nanjing [24]. Chen et al. reported that the proportion of isolates with a CRO MIC $=0.125 \mathrm{mg} / \mathrm{L}$ was $39.4 \%$ in 2007 and $11.3 \%$ in 2012 [25]. The proportion of CRO nonsusceptible isolates $(\mathrm{MIC} \geq 0.125 \mathrm{mg} / \mathrm{L})$ ranged from $22 \%$ to over $48 \%$ in Nanning in 2000-2012 [26], much higher than that detected in Shanghai. Regional differences in gonococcal AMR within a country have also been demonstrated in other countries. In Canada, isolates of $N$. gonorrhoeae from the province of Saskatchewan retained a high prevalence of susceptibility to antibiotics, including CIP and PEN and CRO. Unlike reports of reduced susceptibility to third-generation cephalosporins (CRO or cefixime) from other regions of Canada and the rest of the world, all isolates collected during 2003-2008 from Saskatchewan were fully susceptible to CRO and cefixime [27]. Approximately $7 \%$ of the isolates $(n=23)$ showed a CRO MIC of $0.03-0.06 \mathrm{mg} / \mathrm{L}$, and $93 \%$ of the

Table 3 MDR associated with the CRO nonsusceptible phenotype in $\mathbf{N}$. gonorrhoeae isolates in Shanghai

\begin{tabular}{|c|c|c|c|c|c|c|c|c|}
\hline Year & $\mathbf{N}$ & PEN-CRO & TET-CRO & CIP-CRO & PEN-TET-CRO & TET-CIP-CRO & PEN-CIP-CRO & PEN-TET -CIP-CRO \\
\hline 2001 & 230 & $6.52 \%$ & - & $6.52 \%$ & - & - & $2.17 \%$ & - \\
\hline 2002 & 210 & $7.62 \%$ & - & $9.52 \%$ & - & - & $3.81 \%$ & - \\
\hline 2003 & 302 & $9.60 \%$ & $9.93 \%$ & $10.26 \%$ & $8.94 \%$ & $8.94 \%$ & $8.61 \%$ & $7.95 \%$ \\
\hline 2004 & 166 & $12.05 \%$ & $7.83 \%$ & $12.05 \%$ & $7.83 \%$ & $7.83 \%$ & $12.05 \%$ & $7.83 \%$ \\
\hline 2005 & 83 & $6.02 \%$ & $2.41 \%$ & $0.00 \%$ & $2.41 \%$ & $2.41 \%$ & $6.02 \%$ & $2.41 \%$ \\
\hline 2006 & 128 & $12.50 \%$ & $7.03 \%$ & $13.28 \%$ & $6.25 \%$ & $7.03 \%$ & $12.50 \%$ & $6.25 \%$ \\
\hline 2007 & 200 & $4.50 \%$ & $3.00 \%$ & $5.00 \%$ & $2.50 \%$ & $3.00 \%$ & $4.50 \%$ & $2.50 \%$ \\
\hline 2008 & 71 & $19.72 \%$ & $12.68 \%$ & $19.72 \%$ & $12.68 \%$ & $12.68 \%$ & $19.72 \%$ & $12.68 \%$ \\
\hline 2009 & 157 & $11.64 \%$ & $7.64 \%$ & $11.46 \%$ & $7.64 \%$ & $7.64 \%$ & $11.46 \%$ & $7.64 \%$ \\
\hline 2010 & 119 & $7.56 \%$ & $7.56 \%$ & $7.56 \%$ & $7.56 \%$ & $7.56 \%$ & $7.56 \%$ & $7.56 \%$ \\
\hline 2011 & 135 & $10.37 \%$ & $8.15 \%$ & $10.37 \%$ & $8.15 \%$ & $8.15 \%$ & $10.37 \%$ & $8.15 \%$ \\
\hline 2012 & 99 & $6.06 \%$ & $5.05 \%$ & $7.07 \%$ & $5.05 \%$ & $5.05 \%$ & $6.06 \%$ & $5.05 \%$ \\
\hline 2013 & 109 & $11.93 \%$ & $10.09 \%$ & $13.76 \%$ & $9.17 \%$ & $10.09 \%$ & $11.93 \%$ & $9.17 \%$ \\
\hline
\end{tabular}


isolates $(n=297)$ showed a CRO MIC of $<0.03 \mathrm{mg} / \mathrm{L}$ [27]. In Canada between 2000 and 2009 ( $n=40,875)$, the proportions of PEN, TET or CIP resistant isolates were $11.8 \%, 21.3 \%$ and $13.2 \%$, respectively; and the CRO MIC had shifted from $0.016 \mathrm{mg} / \mathrm{L}$ in 2000 to $0.063 \mathrm{mg} / \mathrm{L}$ in 2009 [28]. In British Columbia (2006-2011), 13\% of isolates (227/1837) showed a CRO MIC of $0.064 \mathrm{mg} / \mathrm{L}$ and $1 \%$ of isolates (27/1837) showed a CRO MIC of $0.125 \mathrm{mg} / \mathrm{L}$ [29]. Indeed, cefixime treatment failure was found in $3.09 \%$ of the investigated cases (9/291) in Toronto between 2010 and 2011 [9]. Taking all of these data into account, it is clear that regional monitoring of $N$. gonorrhoeae AMR is as important as national surveillance programs.

Currently, the recommended therapy for gonorrhea is a single dose of oral or parental third-generation cephalosporin. The doses of CRO used for the treatment of uncomplicated gonorrhea vary among countries; typically 250-500 mg per dose is injected intramuscularly, but in Japan $1 \mathrm{~g}$ is administered intravascularly [14,23,30-34]. A landmark study suggested that increasing the dose of $\mathrm{CRO}$ is associated with a decreased proportion of CRO nonsusceptible $N$. gonorrhoeae isolates in the UK [4]. However, this decrease may not be sustainable [21]. We observed that the proportion of $\mathrm{CRO}$ nonsusceptible isolates transiently increased from 5\% in 2007 when CRO became a recommended drug in China, to over $19 \%$ in 2008, 11\% in 2009 and 13\% in 2013. In Shanghai, CRO at a single low dose $(250 \mathrm{mg}$ ) has been used to treat uncomplicated gonorrhea since 2000, and the proportion of CRO nonsusceptible $N$. gonorrhoeae isolates remains fairly stable. Based on our findings and reports from others in China [24-26], CRO remains an effective antimicrobial for the treatment of uncomplicated gonorrhea. Since CRO nonsusceptible $N$. gonorrhoeae isolates are prevalent in China, a higher dose of CRO (500 mg) is recommended, at least in some areas such as Shanghai, Nanjing and Nanning [24-26].

Detailed data on trends of MDR of $N$. gonorrhoeae in China are limited. However, with the consideration of the high resistant proportions of gonococcal isolates to PEN, TET, and CIP, non-susceptible CRO-associated MDR should not be ignored in China. We observed that over $5 \%$ of isolates were non-susceptible CRO-associated MDR in Shanghai in recent years.

One of the major purposes of antimicrobial susceptibility surveillance is to provide information that may indicate the need for modification of treatment guidelines. The World Health Organization recommended that when the prevalence of resistant gonococci to an antimicrobial agent rises to $5 \%$, the antimicrobial should no longer be used [35]. In China, PEN had been the treatment of choice for gonorrhea until quinolones were introduced in 1993, PEN was removed from the treatment regime in 2000
$[14,15]$. Before 2000, the recommended dose of PEN continually increased because of a progressive decline in susceptibility. Penicillin was removed from the treatment regime 10 years after it was discontinued in 1985 in most regions of the world, simply because of the availability of replacing antibiotics. In 1993, oral fluoroquinolones (CIP and ofloxacin) were recommended for gonorrhea treatment, and at that time CIP resistance was already $15 \%$ in China [12,13]. Four years after (1997), CIP resistance reached over $80 \%$ (100\% in 2000), while the drug remained in the treatment regime until 2007 [14].

Recently potential novel treatment strategies have been explored [6,7]. A strategy of combining older drugs was discussed [36-38]. However, there is no evidence that this combination of older drugs produces synergistic effects. Considering the problems of MDR, this strategy should be used with caution in China. A high prevalence of MDR to almost all currently available drugs in Shanghai limits options for combined therapy. New antibiotics are needed but are largely unavailable [38]. It is therefore worth considering and evaluating SPT as an alternative first line drug for the treatment of uncomplicated urogenital gonorrhea in China. However, SPT is not suitable for the treatment of pharyngeal gonorrhea even though the prevalence of resistance is low in most areas of the world [39]. The MICs of SPT need to be closely monitored, and any signs of increased SPT MIC warrant a thorough investigation and close attention by the policy makers.

The current study has a number of limitations. 1) All of the $N$. gonorrhoeae isolates tested in this study were collected from the Sexually Transmitted Infection Clinic of the SSDH, and only a small number of isolates were tested, accounting for $2.1 \%$ of the total reported cases in 2012 in Shanghai. 2) A lack of demographic and clinical data prevented any comparisons of $N$. gonorrhoeae antimicrobial resistance profiles with patient characteristics; 3) Molecular subtyping of the isolates was not performed. This would provide insight into whether there is clonal spread of resistant isolates and should be the focus of future studies. 4) The agar dilution MIC testing method has an intrinsic error rate of plus/minus one dilution. While using a breakpoint of $0.125 \mathrm{mg} / \mathrm{L}$ for $\mathrm{CRO}$ nonsusceptible isolates, some isolates may actually have MICs of $0.06 \mathrm{mg} / \mathrm{L}$.

\section{Conclusions}

In summary, antimicrobial resistance of $N$. gonorrhoeae to PEN, TET and CIP is highly prevalent in Shanghai. A high proportion of $N$. gonorrhoeae isolates were found to be CRO nonsusceptible ( $\mathrm{MIC} \geq 0.125 \mathrm{mg} / \mathrm{L})$. Plasmidmediated resistance and MDR are also prevalent in China. $N$. gonorrhoeae isolates are susceptible to SPT and this antibiotic needs to be further evaluated as a treatment option for urogenital gonococcal infections. Our findings 
suggest that the CRO dose should be increased to $500 \mathrm{mg}$ in areas with a high prevalence of CRO nonsusceptible isolates. We propose that combination therapy using older antibiotics should be used with caution unless drug synergistic effects can be confirmed. Finally, both national and regional antimicrobial susceptibility surveillance programs are important to inform policy makers in a timely manner on the appropriate modifications of treatment guidelines.

\section{Additional file}

Additional file 1: Figure S1. Resistant proportions of N. gonorrhoeae isolates to CIP, PEN and TET. Closed diamond solid line: CIP resistant; Open square dashed line: PEN resistant; Closed triangle solid line: TET resistant. Surveillance of PEN susceptibility started in 1988, and data to PEN in 1990, 1991 and 1994 were not available. Surveillance of CIP and TET susceptibility started in 1995 (TET) and 1996 (CIP). During 1998-2002, TET was examined only at a concentration of $16 \mathrm{mg} / \mathrm{L}$, and proportions of resistance were unavailable. N: number of isolates tested. R: resistant. Figure S2. Proportions of plasmid-mediated resistance in N. gonorrhoeae. Closed diamond solid line: Penicillinase-producing N. gonorrhoeae (presumed PPNG); Open square dashed line: High level TET resistance N. gonorrhoeae (presumed TRNG); Closed triangle dashed line: presumed PP/TRNG. N: number of isolates tested.

\section{Abbreviations \\ AMR: Antimicrobial resistance; CLSI: Clinical Laboratory Standard Institute; CIP: Ciprofloxacin; CMRNG: Chromosomally-mediated resistant $N$. gonorrhoeae; CRO: Ceftriaxone; EUCAST: European Committee on Antimicrobial Susceptibility Testing; MDR: Multiple drug resistance; MIC: Minimal inhibitory concentration; PEN: Penicillin; PPNG: Penicillinase- producing N. gonorrhoeae; SPT: Spectinomycin; SSDH: Shanghai Skin Disease Hospital; TET: Tetracycline; TRNG: High-level tetracycline-resistant N. gonorrhoeae.}

\section{Competing interests}

The authors declare that they have no competing interests.

\section{Authors' contributions}

WG conceived of and designed the study, participated in data collection and data analysis, and drafted the manuscript. YC participated in the study design and data analysis, performed the statistical analysis and helped to draft the manuscript. YY, LW, WH and YJ carried out sample collection, antimicrobial susceptibility testing and participated in data analysis. All authors read and approved the manuscript.

\section{Acknowledgements}

We thank Dr. Mingmin Liao (University of Saskatchewan, Canada) for assistance in data analysis and in the preparation of the manuscript. This work was supported by the Natural Science Foundation of Shanghai, China (to WM; Grant numbers 06ZR14074, 09DZ1907104 and 02ZB14084) and the Shanghai Health Bureau Science Foundation (to WM; Grant number 1424).

\section{Author details}

${ }^{1}$ Shanghai Skin Disease Hospital, Shanghai, China. ${ }^{2}$ Department of Epidemiology and Community Medicine, Faculty of Medicine, University of Ottawa, Ottawa, Canada.

Received: 19 September 2014 Accepted: 19 December 2014 Published online: 30 December 2014

\section{References}

1. World Health Organization (WHO): Global incidence and prevalence of selected curable sexually transmitted infections - 2008. 20 Avenue Appia, 1211 Geneva 27, Switzerland: WHO Press, World Health Organization; 2012.

2. Qiu X, Jiang N, Gong X: [Reports on syphilis and gonorrhea in China in 2012]. Bulletin Sex Transm Dis 2013, 266:11-21.
3. Jerse $A E$, Deal $C D$ : Vaccine research for gonococcal infections: where are we? Sex Transm Infect 2013, 89(Suppl 4):iv63-iv68.

4. Ison CA, Town K, Obi C, Chisholm S, Hughes G, Livermore DM, Lowndes CM: GRASP Collaborative group: Decreased susceptibility to cephalosporins among gonococci: data from the Gonococcal Resistance to Antimicrobials Surveillance Programme (GRASP) in England and Wales, 2007-2011. Lancet Infect Dis 2013, 13:762-768.

5. Ndowa FJ, Ison CA, Cole MJ, Lusti-Narasimhan M: Gonococcal antimicrobial resistance: challenges for public health control. Sex Transm Infect 2013, 89(Suppl 4):iv3-iv4.

6. Unemo M, Shafer WM: Antimicrobial resistance in Neisseria gonorrhoeae in the $21^{\text {st }}$ century: past, evolution, and future. Clin Microbiol Rev 2014 27:587-613.

7. Ison CA, Deal C, Unemo M: Current and future treatment options for gonorrhoea. Sex Transm Infect 2013, 89(Suppl 4):iv52-iv56.

8. Unemo M, Nicholas RA: Emergence of multidrug-resistant, extensively drugresistant and untreatable gonorrhea. Future Microbio/ 2012, 7:1401-1422.

9. Allen VG, Mitterni L, Seah C, Rebbapragada A, Martin IE, Lee C, Siebert H, Towns L, Melano RG, Low DE: Neisseria gonorrhoeae treatment failure and susceptibility to cefixime in Toronto, Canada. JAMA 2013, 309:163-170.

10. Lewis DA: Global resistance of Neisseria gonorrhoeae: when theory becomes reality. Curr Opin Infect Dis 2014, 27:62-67.

11. Liao M, Bell K, Gu WM, Yang Y, Eng NF, Fu W, Wu L, Zhang CG, Chen Y, Jolly AM, Dillon JA: Clusters of circulating Neisseria gonorrhoeae strains and association with antimicrobial resistance in Shanghai. $J$ Antimicrob Chemother 2008, 61:478-487.

12. Yang Y, Liao M, Gu WM, Bell K, Wu L, Eng NF, Zhang CG, Chen Y, Jolly AM, Dillon JA: Antimicrobial susceptibility and molecular determinants of quinolone resistance in Neisseria gonorrhoeae isolates from Shanghai. J Antimicrob Chemother 2006, 58:868-872.

13. Ye S, Su $X$, Wang $Q$, Yin $Y$, Dai $X$, Sun $H$ : Surveillance of antibiotic resistance of Neisseria gonorrhoeae isolates in China, 1993-1998. Sex Transm Dis 2002, 29:242-245.

14. Wang Q, Zhang G: [Guidelines for diagnosis and treatment of sexually transmitted diseases 2007]. Center for Control of Sexually Transmitted Diseases, Center for Disease Control, China (China CDC). Shanghai, China 200235: Shanghai Sciences and Technology Publishing; 2007.

15. Ministry of Health China: [Guidelines for the treatment of sexually transmitted infections 2000]. Nanjing, China: National Center for Leprosy and STD Control; 2000

16. National Centre for STD Control, China CDC: [Bulletin of Sexually Transmitted Diseases], [Available at: http://www.ncstdc.org/list.asp?id=185; 4 May 2014, date last accessed).

17. Clinical and Laboratory Standard Institute: Performance standards for antimicrobial susceptibility testing: Twenty-fourth informational supplement M100-S24. PA, USA: CLSI Wayne; 2014.

18. European Committee on Antimicrobial Susceptibility Testing (EUCAST) Expert rules 2010 [Available at: http://www.eucast.org/expert_rules. AST to Bacteria Breakpoint Table Version 1.1.; 3 May 2014, date last accessed].

19. Kirkcaldy RD, Kidd S, Weinstock HS, Papp JR, Bolan GA: Trends in antimicrobial resistance in Neisseria gonorrhoeae in the USA: the Gonococcal Isolate Surveillance Project (GISP), January 2006-June 2012. Sex Transm Infect 2013, 89(Suppl 4):iv5-iv10.

20. Martin I, Sawatzky P, Liu G, Allen V, Lefebvre B, Hoang L, Lovgren M, Haldane D, Caeseele PV, Horsman G, Garceau R, Ratnam S, Wong T, Gilmour $\mathrm{M}$ : Antimicrobial susceptibilities and distribution of sequence types of Neisseria gonorrhoeae isolates in Canada: 2010. Can J Microbiol 2013, 59:671-678.

21. Lahra MM, Donovan B, Whiley DM: Decreased susceptibility to cephalosporins among gonococci? Lancet Infect Dis 2014, 14:186.

22. Lahra MM: Australian Gonococcal Surveillance Programme: Annual report of the Australian Gonococcal Surveillance Programme, 2011. Commun Dis Intell 2012, 36:E166-E173.

23. Dillon JA, Trecker MA, Thakur SD: Gonococcal Antimicrobial Surveillance Program Network in Latin America and Caribbean 1990-2011: Two decades of the gonococcal antimicrobial surveillance program in South America and the Caribbean: challenges and opportunities. Sex Transm Infect 2013, 89(Suppl 4):iv36-iv41.

24. Su X, Jiang F, Ge Q, Dai X, Sun H, Ye S: Surveillance of antibmicrobial susceptibilitites in Neisseria gonorrhoeae in Nanjing, China, 1999-2006. Sex Transm Dis 2007, 34:995-999. 
25. Chen SC, Yin YP, Dai XQ, Unemo M, Chen XS: Antimicrobial resistance, genetic resistance determinants for ceftriaxone and molecular epidemiology of Neisseria gonorrhoeae isolates in Nanjing, China. J Antimicrob Chemother 2014, 69:2959-2965.

26. Zhu BY, Yu RX, Yin Y, Chen X, Li W, Dai XQ, Liang M, Gan Q, Huang YJ, Wei JP: Surveillance of antimicrobial susceptibilities of Neisseria gonorrhoeae in Nanning, China, 2000-2012. Sex Transm Dis 2014, 41:501-506.

27. Thakur SD, Starnino S, Horsman GB, Levett PN, Dillon JR: Unique combined penA/mtrR/porB mutations and NG-MAST strain types associated with ceftriaxone and cefixime MIC increases in a 'susceptible' Neisseria gonorrhoeae population. J Antimicrob Chemother 2014, 69:1510-1516.

28. Martin I, Jayaraman G, Wong T, Liu G, Gilmour M: Canadian Public Health Laboratory Network: Trends in antimicrobial resistance in Neisseria gonorrhoeae isolated in Canada: 2000-2009. Sex Transm Dis 2011, 38:892-898.

29. Hottes TS, Lester RT, Hoang LM, McKay R, Imperial M, Gilbert M, Patrick D, Wong T, Martin I, Ogilvie G: Cephalosporin and azithromycin susceptibility in Neisseria gonorrhoeae isolates by site of infection, British Columbia, 2006 to 2011. Sex Transm Dis 2013, 40:46-51.

30. Public Health Agency of Canada: Canadian Guidelines on Sexually Transmitted Infections, 2008 Edition. Public Health Agency of Canada. Ottawa, ON, Canada; 2008.

31. Bignell C, Fitzgerald M: UK national guideline for the management of gonorrhea in adults, 2011. Int J STD AIDS 2011, 22:541-547.

32. Sexual Health Society of Victoria: National Management Guidelines for Sexually Transmitted Infections 7th edition. Melbourne, Australia: Sexual Health Society of Victoria; 2008

33. Centers for Disease Control and Prevention: Update to CDC's Sexually transmitted diseases treatment guidelines, 2010: oral cephalosporins no longer a recommended treatment for gonococcal infections. Morb Mortal Wkly Rep 2012, 61:590-594.

34. Centers for Disease Control and Prevention: Sexually transmited disease treatment guidelines, 2010. Morb Mortal Wkly Rep 2010, 59(RR12):50-52.

35. World Health Organization: Antimicrobial resistance in Neisseria gonorrhoeae Available at WHO/CDS/CSR/DRS/2001.3. 2001. (3 May 2014, date last accessed). http://www.who.int/drugresistance/Antimicrobial_resistance_in_ Neisseria_gonorrhoeae.pdf

36. Pereira R, Cole MJ, Ison CA: Combination therapy for gonorrhoea: in vitro synergy testing. J Antimicrob Chemother 2013, 68:640-643.

37. Lusti-Narasimhan M, Pessoa-Silva CL, Temmerman M: Moving forward in tackling antimicrobial resistance: WHO actions. Sex Transm Infect 2013, 89(Suppl 4):iv57-iv59.

38. Ndowa F, Lusti-Narasimhan $\mathrm{M}$, Unemo M: The serious threat of multidrugresistant and untreatable gonorrhoea: the pressing need for global action to control the spread of antimicrobial resistance, and mitigate the impact on sexual and reproductive health. Sex Transm Infect 2012, 88:317-318.

39. Gil-Setas A, Navascués-Ortega A, Beristain X: Spectinomycin in the treatment of gonorrhoea. Euro Surveill, 2010; 15:pii = 19568. Available at http://www. eurosurveillance.org/ViewArticle.aspx?Articleld=19568. (3 May 2014, date last accessed).

doi:10.1186/s12879-014-0731-9

Cite this article as: Gu et al:: Twenty-five-year changing pattern of gonococcal antimicrobial susceptibility in Shanghai: surveillance and its impact on treatment guidelines. BMC Infectious Diseases 2014 14:731.

\section{Submit your next manuscript to BioMed Central and take full advantage of:}

- Convenient online submission

- Thorough peer review

- No space constraints or color figure charges

- Immediate publication on acceptance

- Inclusion in PubMed, CAS, Scopus and Google Scholar

- Research which is freely available for redistribution

Submit your manuscript at www.biomedcentral.com/submit
C Biomed Central 